Sección Tres: Experiencias, reseñas, debates e informes

Radiografía de la innovación educativa en el Siglo XXI

\title{
Decir algo sociológico sobre la evaluación en la universidad y la investigación científica ${ }^{1}$
}

Saying something sociological about evaluation in university and scientific research

\author{
Davide Borrelli \\ Universidad degli Studi Sour \\ Orsola Benincasa Napoli (Italy) \\ davide.borrelli@unisob.na.it
}

\section{Debates}

No quiero hablar en esta ocasión de los efectos perversos, de las devastadoras consecuencias sistémicas o de los diferentes defectos metodológicos de los dispositivos de evaluación de la calidad de universidad y de la investigación que se aplican en Europa (véanse Pinto 2012, Borrelli 2015, Collard 2018).

Después de todo, una disputa técnica tiende a entrar en el juego del actual dispositivo porque, absorbida en su código, corre el riesgo de diluir cualquier radicalidad, es decir, de no atacar las raíces del problema. La mía no pretende ser una crítica en el código, sino una crítica del código. Una crítica radical, de hecho, que se deriva de un malestar vivido, que imagino común a muchos de los investigadores. Una crítica similar a las "pruebas existenciales” de las que habla Luc Boltanski: “no solo constituyen una 'prueba' en el sentido de 'test', sino [...] también 'prueba' en el sentido de algo de lo que estamos 'probados' y que por lo tanto suscita una forma de sufrimiento, al menos psíquico, algo que nos toca de cerca” (2014, p. 160).

Me pone a prueba y me toca muy de cerca que desde hace años se hayan limitado recursos a escuelas y universidades, cuando en cambio deberían ser los pilares de cada país civilizado. Y me pone a prueba y me asalta la indignación, cada vez que (respecto a estos recortes financieros crónicos), escucho que el verdadero problema de la universidad italiana habría sido la falta de un sistema de evaluación serio. Del mismo modo me toca de cerca ver penalizado por los algoritmos de primas a universidades que ya están en gran medida en

${ }^{1}$ Recibido: 20/02/2020 Evaluado: 23/02/2020 Aceptado: 23/02/2020 
desventaja, ya que operan en contextos socioeconómicos desfavorecidos. Me toca de cerca y me irrita la empalagosa narración meritocrática utilizada para legitimar éticamente la desigualdad, es decir, para culpar a las propias víctimas de la condición que padecen. Me afecta de cerca y me preocupa la barbaridad de establecer los criterios para una distribución de fondos ex post, transformando por ejemplo de la cuna a la tumba del estado de derecho a Italia (y me preocupa que casi nadie se preocupe por ello hasta ahora). Me toca muy de cerca y lamento que, mientras somos rehenes del valor de los umbrales, las revistas con impacto y toda la basura de los añadidos medios de distracción masiva, que el efecto certificador de la formalización final de una plaza, sea visible años después de iniciar el proceso (en el caso de Italia de cuatro a nueve años). Me toca de cerca y me parece francamente lamentable que haya unas vías aceleradas en ciertos lugares de excelencia (principalmente concentrados en ciertas áreas del país), y en cambio vías muertas en otros, donde la acreditación sirve nada más que para un principio de consolación narcótica. Finalmente, me toca de cerca y despierta una sensación de dolor y vergüenza infinita ver nuestra dignidad profesional, nuestra autonomía científica y nuestra propia inteligencia de sociólogos pisoteadas en el nombre de la evaluación.

Y es precisamente como sociólogo y dirigido a sociólogos como me gustaría abordar el tema de lo que se llama "nueva evaluación" para distinguirla de los tradicionales sistemas de reflexión en uso en comunidades científicas. Este dispositivo nos desafía a todos como comunidad a decir algo sociológico, como en la famosa escena de la película Aprile (1998) de Nanni Moretti, que invita al presidente Massimo D’Alema a decir algo de izquierdas en un debate político con Berlusconi.

Lo primero que hay que decir es que nos enfrentamos a un giro gubernamental de las prácticas de evaluación: Guy Neave (2012, p. 49) habló del “estado de la evaluación”, aclarando que, si en el pasado el estado ejercía la función de tutor de la autonomía de la universidad, hoy funciona en lugar de eso "como 'garante universitario para el mercado"”. Y no se trata tanto de una colonización endógena, sino que de la asunción endógena y consciente por parte del gobierno de la universidad, del nómos de la competición de mercado. Y no sabría si por una adhesión convencida o un conformismo oportunista.

Pero, ¿realmente vale la pena una universidad alineada e isomorfa con el entorno en el que opera? "Desde un punto de vista ecológico, - explicó Neil Postman (1979) - nada es bueno en sí mismo [...] Lo que hace que algo sea bueno o útil es la existencia de una fuerza opuesta que lo mantiene bajo control”. La instrucción debería funcionar como un termostato para la sociedad: su virtud sería desencadenar fuerzas opuestas y contracorrientes, es decir, en palabras de Postman, “ofrecer un contraargumento, la otra cara de la moneda”. En resumen, la universidad tiene un papel ecológico más que teleológico, es decir, no promueve un objetivo definido, sino que mantiene un equilibrio plural de diferencias en el sistema al tiempo que conserva oportunidades de "contraargumentos".

Decir algo sociológico sobre la nueva evaluación significa, en primer lugar, desmitificar su carácter ritual de manifestación de la verdad, de una verdad de poder excesivamente exhibida y justificada de manera pretenciosa (Foucault 2016). Significa devolver el proceso de toma de decisiones al terreno de la deliberación democrática y salir del hechizo del "realismo 
capitalista” (Fisher 2009), es decir, del there is no alternative que produce opciones y negar que sean tales, de acuerdo con un probado guion político de despolitización.

Permítanme ser claro, renunciamos a decir algo sociológico cada vez que nos sometemos al mantra de la inevitabilidad, sabiendo bien que en el mundo de la política nunca hay realmente nada inevitable. Cuando la sociología queda atrapada por los cantos de sirenas de una "nueva evaluación”, se convierte en el enterrador de sí mismo porque respalda un hábito mental que deslegitima su estado epistémico y su razón de ser. Entonces, la primera víctima de la "nueva evaluación” es la idea misma de una ciencia social y política. Tomar decisiones sobre la base de la evaluación de los méritos individuales, significa liquidar la percepción del horizonte social en el que tienen lugar los fenómenos humanos; es decir, olvidar que el individuo no es una entidad abstracta (como el homo oeconomicus), sino que se define por su contexto y su historia. Hacerlo sobre la base de un dispositivo que se afirma neutral y objetivo implica olvidar la naturaleza intrínsecamente política de cada decisión. Basta pensar en el contexto en el que fue concebido el primer ejercicio de evaluación superior de la investigación científica: en el Reino Unido por Margaret Thatcher, para comprender la razón gubernamental de la que derivan el énfasis en la calidad universitaria, la competición como estrategia para lograrla y la evaluación como herramienta para medirla. Una de las frases más famosas de Thatcher, que expresa una visión del mundo perjudicial para las ciencias sociales, es que "no existe la sociedad, solo hay individuos". Atención, la navaja de Occam es peligrosa para la sociología: solo un momento y te encuentras epistemológicamente mutilado.

Decir algo sociológico sobre la “nueva evaluación” también significa darse cuenta de que lo que sucede en nuestra universidad nos habla del espíritu de la actualidad y la involución postdemocrática de nuestra sociedad (Crouch 2004). Las políticas universitarias actuales están perfectamente enmarcadas en una forma de dominación centrada en la idea del "gobierno técnico, prerrogativa del supuesto conocimiento experto. En realidad, en lugar de "gobiernos técnicos”, son verdaderas "técnicas de gobierno" que esterilizan la dialéctica política. En nombre de la competitividad, lo posible se reduce y las diferencias se aminoran. $\mathrm{Y}$, aunque es dudoso que los dogmas neoliberales realmente puedan mantenernos fuera de la crisis económica, es cierto que una alarmante crisis democrática nos está precipitando. Es emblemático de esta progresiva desmocratización de la sociedad (por citar a Wendy Brown, 2015), por ejemplo, el uso de la palabra "gobernanza" en lugar de "gobierno". Lo mismo ocurre con el llamado proceso de Bolonia que inició las reformas de los sistemas de educación superior en Europa: la palabra "proceso" oculta su naturaleza de elección política disfrazándola de intervención administrativa.

Los académicos (y nosotros los sociólogos entre ellos) nos dejamos inscribir en este proyecto posdemocrático de sociedad y de universidad sin dificultades particulares, de hecho a veces con una diligencia demasiado entusiasta que no parezca sospechosa. Una especie de síndrome de Estocolmo, se podría decir.

Sin embargo, sabemos bien que la "nueva evaluación” produce la normalización del conocimiento y el "dopaje bibliométrico en masa" (Baccini et alii, 2019), ya que todos estamos a favor de "escribir y publicar a expensas de leer y estudiar" (Mats Alvesson et alii, 2017). Tampoco nos escapa que la nueva evaluación es el brazo de una "nueva razón del 
mundo" (Dardot y Laval, 2013), que consiste en "la generalización de la competencia corno norma de conducta y de la empresa como modelo de subjetivación” (p. 15).

Porque la ciencia y la producción científica tienen una extraña alianza con la tecnología hoy. Pueden (Pérez-González y Ribón, 2019) avanzar, sufrir una regresión, porque el conocimiento es ahora otro tipo de dominación, inédita en la historia de la humanidad; un tipo de poder inducido, de una capacidad de maniobra. Si siguiese otra lógica, que no fuera la de la jerarquización, podría extraerse del conocimiento su capacidad de análisis y de crítica.

Esta es la única forma de explicar la afirmación paradójica que a menudo escuchamos, es decir que incluso una mala evaluación sería preferible a la ausencia de evaluación. Si reflexionamos en ello, significa que no se evalúa realmente para fines científicos, sino para gobernar las conductas de acuerdo con un diseño de ortopedia cognitiva que tiene como objetivo cambiar el espíritu del investigador para transformarlo en un verdadero emprendedor de la investigación.

Garantía de calidad, meritocracia, excelencia, clasificación: ha habido una inflación vertiginosa de neo-lenguaje mitológico y gerencial en los últimos años. Aprendimos de Roland Barthes (2012) que el discurso mitológico sirve para naturalizar y o pacificar puntos de vista que son contingentes $y$, por lo tanto, discutibles.

Consideremos, por ejemplo, el mito de la meritocracia. Las reiteradas promesas de castigar a los llamados "holgazanes" y premiar a los presuntos merecedores han demostrado ser una cortina de humo para cubrir los recortes financieros. El concepto mismo de meritocracia es el resultado de un fraude semántico sin escrúpulos. Cuando Michael Young (1958) lo propuso, este término tenía un significado negativo y distópico. Hoy, sin embargo, se usa mitológicamente como si fuera la panacea para una sociedad mejor y más justa.

Otro mito hoy es el llamado premio de financiación. Estos premios a las supuestas estructuras dignas son un caballo de Troya; son como los trozos de carne que los ladrones arrojan a los perros guardianes para poder robar una casa sin ser molestados. Nuestra casa común, la universidad, durante años. Y creer que los sistemas de recompensas por evaluación sirven para garantizar su calidad, es como pensar que la función de los ladrones es alimentar a nuestros perros.

Contra estas manipulaciones, y concluyo, hace falta una resistencia que deconstruya las mitologías de hoy sobre la universidad. Basta de ius murmurandi en los pasillos, practiquemos el "coraje de la verdad” (Foucault 2014) y organicémonos todos juntos para liberarnos de nuestras cadenas mentales. Hoy, como en la época de Etienne de la Boétie, autora del famoso Discurso de la servidumbre voluntaria, sorprende "cómo es posible que tantos hombres ... a veces tengan un tirano, que no tiene otro poder que el que ellos mismos le otorgan”. La salida habría y Etienne de la Boétie nos lo mostró: "Podrías deshacerte del tirano de ti mismo si intentas no [digo] deshacerte de él, sino solo querer hacerlo. Decide no servir más y serás libre”. 


\section{Referencias}

Alvesson, Mats, Gabriel, Yannis y Paulsen, R. (2017). Return to Meaning: a Social Science with Something to Say, Oxford University: Oxford.

Baccini, A., De Nicolao, G. y Petrovich, E. (2019). “Citation gaming induced by bibliometric evaluation: A country-level comparative analysis”, PlosOne, disponible en https://cutt.ly/gtrO9jT

Barthes, R. (2012). Mitologías, Biblioteca Nueva: Madrid.

Boltanski, L. (2014). Della critica: Compendio di sociologia dell'emancipazione, Rosenberg \& Sellier: Torino.

Borrelli, D. (2015). Contro l'ideologia della valutazione: l'Anvur e l'arte della rottamazione dell'università, Jouvence: Milano.

Brown, W. (2015). Undoing the demos: neoliberalism's stealth revolution, Zone Books: New York.

Collard, D. (2018). Le travail, au-delà de l'évaluation: normes et résistances, Éditions érès: Toulouse.

Crouch, C. C. (2004). Posdemocracia, Taurus: Orense.

Dardot, P. y Laval, C. (2013). La nueva razón del mundo: ensayo sobre la sociedad neoliberal, Gedisa: Barcelona.

Fisher, M. (2009). Capitalist realism: is there no alternative?, Zero Books: Ropley.

Foucault, M. (2014). El coraje de la verdad: el gobierno de sí y de los otros, II. Curso del Collège de France (1983-1984), Akal: Madrid.

Foucault, M. (2016). Del gobierno de los vivos. Curso del Collège de France (1979-1980), Akal: Madrid.

La Boétie, É. de (2019). Discurso de la servidumbre voluntaria, Editorial Trotta, Madrid.

Neave, G. (2012). The Evaluative State, Institutional Autonomy and Re-engineering Higher Education in Western Europe, Palgrave MacMillan: New York.

Pérez-González, A-Beatriz y Ribón, M. A. (2019). Consolidación del lenguaje visual en la educación, en Nuevos enfoques para la docencia universitaria. Madrid: Ediciones Pirámide 
Pinto, V. (2012). Valutare e punire. Una critica della cultura della valutazione, Napoli: Cronopio,

Postman, N. (1979). Teaching as a Conserving Activity, New York: Delacorte Press.

Young, Michael (1964). El triunfo de la meritocracia 1870-2034: ensayo sobre la educación y la igualdad, Madrid: Tecnos. 